\title{
Measurement of the $K_{S}$ lifetime and CPT symmetry tests in the neutral kaon system with quantum interferometry at KLOE
}

\author{
Marco DREUCCl*i \\ Istituto Nazionale di Fisica Nucleare, Frascati
}

E-mail: marco.dreucci@lnf.infn.it

A $\phi$-factory offers the possibility to select pure kaon beams. Neutral kaons from $\phi \rightarrow K_{S} K_{L}$ are produced in a pure $J^{P C}=1^{--}$quantum state. Detection of a kaon at large (small) times tags a $K_{S}\left(K_{L}\right)$. This allows to perform precise measurement of kaon properties, as for example lifetime, and to study time evolution of neutral kaon system. The distribution of $\Delta t$, the difference between the two neutral kaon decay times, where both kaons decay into a $\pi^{+} \pi^{-}$pair, provides unique opportunities for testing quantum mechanics and CPT symmetry. Using the full 20042005 data sample $\left(L=1.5 \mathrm{fb}^{-1}\right)$ collected with the KLOE detector at DAФNE, the Frascati $e^{+} e^{-}$ collider, we update results of quantum mechanics and CPT invariance tests. We are finalizing the determination $K_{S}$ lifetimes using about $1 / 3$ the whole KLOE data set. The proper time distribution of $K_{S} \rightarrow \pi^{+} \pi^{-}$decays provides a competitive measurement of $K_{S}$ meson lifetime.

European Physical Society Europhysics Conference on High Energy Physics, EPS-HEP 2009, July 16 - 222009

Krakow, Poland

\footnotetext{
* Speaker.

$\dagger$ On behalf of KLOE collaboration: F. Ambrosino, A. Antonelli, M. Antonelli, F. Archilli, P. Beltrame, G. Bencivenni, C. Bini, C. Bloise, S. Bocchetta, F. Bossi, P. Branchini, G. Capon, D. Capriotti, T. Capussela, F. Ceradini, P. Ciambrone, E. De Lucia, A. De Santis, P. De Simone, G. De Zorzi, A. Denig, A. Di Domenico, C. Di Donato, B. Di Micco, M. Dreucci, G. Felici, S. Fiore, C. Gatti, P. Gauzzi, S. Giovannella, E. Graziani, M. Jacewicz, V. Kulikov, G. Lanfranchi, J. Lee-Franzini, M. Martini, P. Massarotti, S. Meola, S. Miscetti, M. Moulson, S. Muller, F. Murtas, M. Napolitano, F. Nguyen, M. Palutan, A. Passeri, V. Patera, P. Santangelo, B. Sciascia, A. Sibidanov, T. Spadaro, L. Tortora, P. Valente, G. Venanzoni, R. Versaci.
} 


\section{Quantum mechanical coherence test}

The $J^{P C}=1^{--}$two neutral kaon state can be written as

$$
\frac{N}{\sqrt{2}}\left(\left|K_{S}, \mathbf{p}\right\rangle\left|K_{L},-\mathbf{p}\right\rangle-\left|\mathbf{K}_{\mathbf{L}}, \mathbf{p}\right\rangle\left|\mathbf{K}_{\mathbf{S}},-\mathbf{p}\right\rangle\right)
$$

Time evolution obeys a Schrödinger-like equation with $H=M-i \Gamma / 2$, and the intensity decay into final states $f_{1}$ and $f_{2}$, respectively at the time $t_{1}$ and $t_{2}$, can be expressed as follows:

$$
I\left(f_{1}, t_{1} ; f_{2}, t_{2}\right) \sim\left|a_{1 S} a_{2 L}\right|^{2}+\left|a_{1 L} a_{2 S}\right|^{2}-2\left(1-\zeta_{S L}\right) \Re\left\{\left(a_{1 S} a_{2 L}\right)^{*}\left(a_{1 L} a_{2 S}\right)\right\}
$$

where $\zeta_{S L}$ is an ad hoc decoherence parameter and $a_{i S(L)}=\left\langle f_{i}|T| K_{S(L)}\left(t_{i}\right)\right\rangle$, where $i=1,2$. If both kaons decay in the same final states, $f_{1}=f_{2}=\pi^{+} \pi^{-}$, integrating over one time decay for a fixed $\Delta t=\left|t_{2}-t_{1}\right|$ gives the intensity decay distribution, $I(\Delta t)$, where $\Delta t=\left|t_{2}-t_{1}\right|$

$$
I(\Delta t) \sim \exp \left(-\Gamma_{L} \Delta t\right)+\exp \left(-\Gamma_{S} \Delta t\right)-2\left(1-\zeta_{S L}\right) \exp \left[-\left(\Gamma_{L}+\Gamma_{S}\right) \Delta t / 2\right] \cos (\Delta m \Delta t)
$$

Initial symmetry requires no events at the same time, i.e., $\Delta t=0$; any violation could be an indication of $\zeta_{S L} \neq 0$. Using Eq.1.3 to fit data distribution (Fig.1, left), where $\Gamma_{S}, \Gamma_{L}$ and $\Delta m$ are fixed at the PDG values, KLOE, analizing about $1.5 \mathrm{fb}^{-1}$, obtains [1]

$$
\zeta_{S L}=\left(0.3 \pm 1.8_{\text {stat }} \pm 0.6_{\text {syst }}\right) \times 10^{-2} ; \quad \zeta_{0 \overline{0}}=\left(1.4 \pm 9.5_{\text {stat }} \pm 3.8_{\text {syst }}\right) \times 10^{-7}
$$

compatible with the prediction of quantum mechanics, i.e., $\zeta_{S L}=\zeta_{0 \overline{0}}=0$, and no decoherence effect. The result of the second of Eq.1.4 is obtained when expressing initial state in the basis $K^{0} \bar{K}^{0}$ and its very high accuracy is due to the CP suppression present in the specific decay channel. These results are a very impressive improvement with respect to the previous results ([2],[3]).
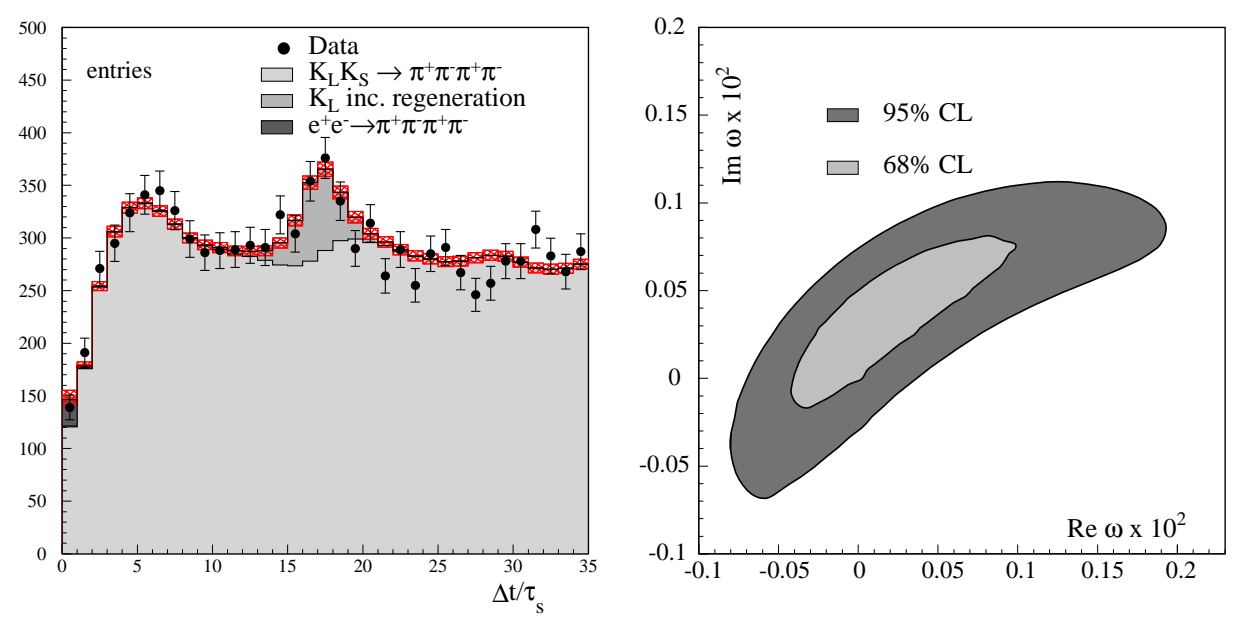

Figure 1: Left: fit to $I(\Delta t)$ distribution ; right: $1-\sigma$ ans $2-\sigma$ contour to the $\omega$ parameter. 


\section{Decoherence and CPT violation due to QG effects}

In presence of decoherence and CPT violation induced by quantum gravity (QG) (CPT operator ill-defined) the definition of the particle-antiparticle states could be modified. This in turn could induce a breakdown of the correlations imposed by Bose statistics (EPR correlations) to the initial kaon state [4], making possible to have

$$
|i\rangle \sim\left(\left|K^{0}\right\rangle\left|\bar{K}^{0}\right\rangle-\left|\bar{K}^{0}\right\rangle\left|K^{0}\right\rangle\right)+\omega\left(\left|K^{0}\right\rangle\left|\bar{K}^{0}\right\rangle+\left|\bar{K}^{0}\right\rangle\left|K^{0}\right\rangle\right)
$$

with $\omega$ at most $|\omega| \sim 10^{-3}$. The maximum sensitivity to $\omega$ is expected for $f_{1}=f_{2}=\pi^{+} \pi^{-}$. Fitting the intensity decay distribution $I(\Delta t)\left(1 \mathrm{fb}^{-1}\right)$ gives [1]

$$
\mathfrak{R} \omega=\left(-1.6_{-2.1 \text { stat }}^{+3.0} \pm 0.4_{\text {syst }}\right) \times 10^{-4} ; \mathfrak{I} \omega=\left(-1.7_{-3.0 \text { stat }}^{+3.3} \pm 1.2_{\text {syst }}\right) \times 10^{-4}
$$

with $|\omega|<10^{-3}$ at $95 \%$ CL (Fig.1, right).

\section{3. $K_{S}$ lifetime}

The main problem with measuring the $K_{S}$ lifetime is that its value, about $90 \mathrm{ps,} \mathrm{is} \mathrm{much} \mathrm{smaller}$ than KLOE detector time resolution, which is about $0.5 \mathrm{~ns}$. This can be balanced by the very large statistics available. In this analysis we use about 25 million $K_{S} \rightarrow \pi^{+} \pi^{-}$decay events. Lifetime is obtained via a fit to the proper time distribution. After selecting two tracks originating from the
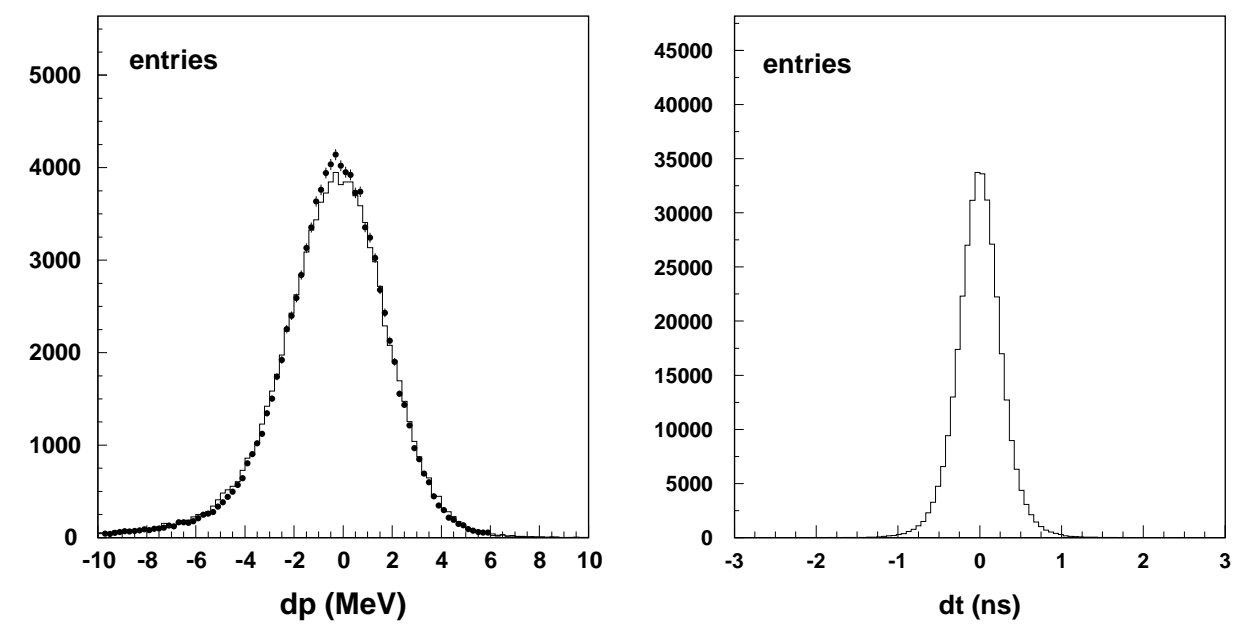

Figure 2: Left: data (dots) and Monte Carlo (line) comparison for the varaiable $d p=p_{\pi \pi}-p_{K}$; right: final proper time resolution.

interaction point (IP) region with an invariant mass close to the kaon mass, using the kaon line of flight we are able to reconstruct event by event the IP with a resolution of about $2 \mathrm{~mm}$. In KLOE a redundant information on the kaon mementum is available: from pions $\left(p_{\pi \pi}\right)$ and from $\sqrt{s}$ and the knowledge of kaon line of fligth $\left(p_{K}\right)$. In Fig. 2 left is shown a comparison between data and Monte Carlo (MC). To improve proper time resolution tight cuts are applied on kaon invariant mass (about $2 \sigma$ ), pions and kaon polar angle. After this selection, the good resolution on $K_{S}$ decay vertex allows 

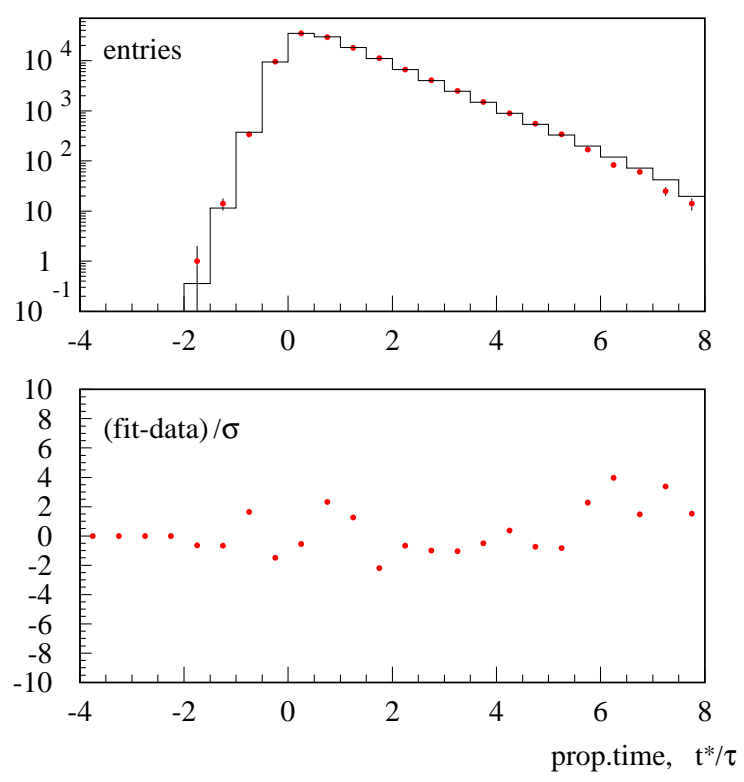

Figure 3: A fit example with $\chi^{2} /$ dof $=8 / 14$.

\begin{tabular}{|lc|} 
source & $t^{*} / \tau_{S}$ fractional value $\times 10^{4}$ \\
\hline selection cuts & 3.3 \\
$\cos \theta_{K}, \mathrm{FV}$ cut & 5.7 \\
kaon mass & 0.4 \\
fit range & 5.0
\end{tabular}

Table 1: Systematic error contributions.

to correct its position along kaon line of flight using Monte Carlo. Finally a geometrical fit, where the kaon direction is assumed correct, but the IP can be moved along the beam $(z)$ axis, allows to reach a resolution of about $0.4 \mathrm{~ns}$ (Fig.2 right). Final resolution, flat in proper time, is about $13 \%$. Because of the different time resolution in different regions of the detector we perform a $\left\{\theta_{K}, \phi_{K}\right\}$ binned analysis, for a total of 270 independent fits. Fit parameters are lifetime, time resolutions (we use two gaussians) and the two gaussian contribution ratio. The fit region is $[-2,+7] t^{*} / \tau$. In Fig. 3 a fit example is shown. The distribution of $\chi^{2}$ from each fit is compatible with the expected distribution. The evaluation of systematics is made with the variation cuts method. In Table 1 are shown contributions from different sources. The preliminary KLOE result for $K_{S}$ lifetime is

$$
\tau_{S}=\left(89.56 \pm 0.03_{\text {stat }} \pm 0.07_{\text {syst }}\right) \mathrm{ps}
$$

in agreement with recent measurements.

\section{References}

[1] A. Di Domenico et al., Journal of Physics 171, 012008 (2009) 
[2] R.A. Bertlmann et al., Physical Review D 60, 114032 (1999)

[3] A. Go et al., Phys.Rev.Lett. 99, 131802 (2007)

[4] J. Bernabeu et al., Phys.Rev.Lett. 92, 131601 (2004) 